\title{
The mutation spectrum in RECQL4 diseases
}

H Annika Siitonen ${ }^{1}$, Jenni Sotkasiira ${ }^{1}$, Martine Biervliet ${ }^{2}$, Abdelmadjid Benmansour ${ }^{3}$, Yline Capri ${ }^{4}$, Valerie Cormier-Daire ${ }^{5}$, Barbara Crandall ${ }^{6}$, Katariina Hannula-Jouppi ${ }^{7}$, Raoul Hennekam ${ }^{8,9}$, Denise Herzog ${ }^{10}$, Kathelijn Keymolen ${ }^{11}$, Marita Lipsanen-Nyman ${ }^{12}$, Peter Miny ${ }^{13}$, Sharon E Plon ${ }^{14}$, Stefan Riedl ${ }^{15}$, Ajoy Sarkar ${ }^{16}$, Fernando R Vargas ${ }^{17}$, Alain Verloes $^{18}$, Lisa L Wang ${ }^{19}$, Helena Kääriäinen ${ }^{1,20}$ and Marjo Kestilä*,1

\footnotetext{
${ }^{1}$ Department of Molecular Medicine, National Public Health Institute and FIMM, Institute for Molecular Medicine Finland, Helsinki, Finland; ${ }^{2}$ Department of Medical Genetics, University of Antwerp, Antwerp, Belgium; ${ }^{3}$ General Pediatrician, 35, rue de Mostaganem, Oran, Algeria; ${ }^{4}$ Department of Medical Genetics and INSERM, CIC501, HôtelDieu, Clermont Ferrand, France; ${ }^{5}$ Department of Medical Genetics and INSERM U 781, Hopital NeckerEnfants Malades, Paris, France; ${ }^{6}$ Departments of Pediatrics and Psychiatry, David Geffen School of Medicine, University of California, Los Angeles, CA, USA; ${ }^{7}$ Department of Medical Genetics, University of Helsinki, Helsinki, Finland; ${ }^{8}$ Clinical and Molecular Genetics Unit, Institute of Child Health, London, UK; ${ }^{9}$ Department of Pediatrics, Academic Medical Center, Amsterdam, The Netherlands; ${ }^{10}$ Department of Pediatrics, University of Montreal, Montreal, Canada; ${ }^{11}$ Center Medical Genetics, UZ Brussel, Brussels, Belgium; ${ }^{12}$ Children's Hospital, University of Helsinki, Helsinki, Finland; ${ }^{13}$ Division of Medical Genetics, University Children's Hospital, Basel, Switzerland; ${ }^{14}$ Departments of Human and Molecular Genetics and Pediatrics, Section of Hematology/Oncology, Texas Children's Cancer Center, Baylor College of Medicine, Houston, TX, USA; ${ }^{15}$ St Anna Children's Hospital, Vienna, Austria; ${ }^{16}$ Clinical and Molecular Genetics Unit, Institute of Child Health and Great Ormond Street Hospital for Children, UCL, London, UK; ${ }^{17}$ Genetics Division, Instituto Nacional de Câncer, Universidade Federal do Estado do Rio de Janeiro, Rio de Janeiro, Brazil; ${ }^{18}$ Department of Genetics, APHP Robert Debré University Hospital, Paris, France; ${ }^{19}$ Department of Pediatrics, Section of Hematology/ Oncology, Texas Children's Cancer Center, Baylor College of Medicine, Houston, TX, USA; ${ }^{20}$ Department of Medical Genetics, University of Turku, Turku, Finland
}

Mutations in the RECQL4 gene can lead to three clinical phenotypes with overlapping features. All these syndromes, Rothmund-Thomson (RTS), RAPADILINO and Baller-Gerold (BGS), are characterized by growth retardation and radial defects, but RAPADILINO syndrome lacks the main dermal manifestation, poikiloderma that is a hallmark feature in both RTS and BGS. It has been previously shown that RTS patients with RECQL4 mutations are at increased risk of osteosarcoma, but the precise incidence of cancer in RAPADILINO and BGS has not been determined. Here, we report that RAPADILINO patients identified as carriers of the c.1390 + 2delT mutation (p.Ala420_Ala463del) are at increased risk to develop lymphoma or osteosarcoma (6 out of 15 patients). We also summarize all the published RECQL4 mutations and their associated cancer cases and provide an update of 14 novel RECQL4 mutations with accompanying clinical data. European Journal of Human Genetics (2009) 17, 151-158; doi:10.1038/ejhg.2008.154; published online 20 August 2008

Keywords: RECQL4; RAPADILINO syndrome; Rothmund-Thomson syndrome; Baller-Gerold syndrome; osteosarcoma; lymphoma

*Correspondence: Dr M Kestilä, Department of Molecular Medicine, National Public Health Institute and FIMM, Institute for Molecular Medicine Finland, Biomedicum Helsinki, Helsinki 00290, Finland.

Tel: + 3589474487 23; Fax: + 358947448 480;

E-mail: marjo.kestila@ktl.fi

Received 4 March 2008; revised 23 July 2008; accepted 24 July 2008; published online 20 August 2008 


\section{Introduction}

Mutations in the RECQL4 gene are known to cause three different autosomal recessive syndromes. These mutations were first found in a subgroup of Rothmund-Thomson syndrome (RTS, MIM 268400) patients ${ }^{1}$ and subsequently in patients diagnosed with RAPADILINO syndrome (MIM $266280)^{2}$ as well as in some Baller-Gerold syndrome (BGS, MIM 218600) patients. $^{3}$ Before this study, a total of 35 RECQL4 mutations have been published (Table 1, Supplementary Table 1$).{ }^{1-12}$

In 1868, Rothmund ${ }^{22}$ described patients with poikiloderma, growth retardation and juvenile cataracts. In 1936, Thomson $^{23}$ published a clinical description of patients with poikiloderma and growth retardation without juvenile cataracts. Later, Taylor ${ }^{24}$ suggested that these patients may have related disorders and coined the eponym RTS. In addition to the features described above, other clinical features also include skeletal dysplasias, gastrointestinal disturbances, sparse scalp hair and sparse eyebrows or lashes. ${ }^{25}$ Thus far, over 250 RTS patients have been reported in the literature and re-evaluation of the distinctive features of RTS has led to the creation of two subclasses. RothmundThomson syndrome type I is defined by the characteristic poikiloderma and lack of RECQL4 mutations. This group also includes patients with juvenile cataracts. RothmundThomson syndrome type II patients have poikiloderma as well, but in addition they have a high risk of osteosarcoma, which seems to be related to mutations in RECQL4. ${ }^{9}$ In molecular studies, which focused on patients with the clinical diagnosis of RTS RECQL4 mutations were found in $\sim 40-66 \%$ of RTS cases. ${ }^{1,9}$ Although there are a significant number of RTS patients without known mutations, no other causative genes have yet been identified for RTS.

RAPADILINO syndrome was first described by Kääriäinen et al in 1989. ${ }^{17}$ These patients have overlapping features with RTS patients, namely intrauterine and postnatal growth retardation and bone malformations, especially radial defects, such as hypoplasia and aplasia of thumbs and radius. However, poikilodermatous rash has never been observed in RAPADILINO patients. In addition, patients with RAPADILINO syndrome do not have alopecia or the absence of eyebrows and eyelashes, features that are usually encountered in RTS. Thus far, 15 RAPADILINO patients have been identified in Finland where RAPADILINO syndrome is overrepresented because of the enrichment of a founder mutation (c.1390+2delT/p.Ala420_ Ala463del). ${ }^{2}$ As only a few RAPADILINO cases have been described in other populations, ${ }^{26,27}$ RAPADILINO syndrome is considered to be genetically homogenous.

Baller-Gerold syndrome is genetically heterogeneous, and mutations have been identified in the RECQL4, FGFR2 and TWIST genes in patients with the BGS phenotype. $^{3,28,29}$ Baller-Gerold syndrome has overlapping clinical features with RTS and RAPADILINO, but the narrow definition of BGS is craniosynostosis with radial aplasia. ${ }^{30}$
However, craniosynostosis has also been reported in patients diagnosed as RTS and, for instance, the London Medical Databases (www.lmdatabases.com/) list it as one of the features of both RTS and BGS syndromes. On account of the phenotypic and genotypic overlap between BGS and other syndromes the existence of BGS as a separate entity has been debated. ${ }^{30,31}$

RECQL4 belongs to the RecQ gene family of DNA helicases, other members being RECQL1, BLM, WRN and RECQL5. ${ }^{32}$ The function of these helicases is to maintain the genomic stability that is needed in all eukaryotic organisms. ${ }^{33,34}$ In addition, defects in the BLM and WRN genes lead to severe inherited diseases (Bloom syndrome (MIM 210900) and Werner syndrome (MIM 277700)) having overlapping features with RECQL4 syndromes, such as cancer predisposition, growth retardation and developmental abnormalities. ${ }^{35}$

The strongest expression of RECQL4 in human tissues was observed in the thymus and testis ${ }^{32}$ whereas the most prominent expression was seen in developing bone, cartilage and intestine when studying expression in the mouse embryos (E15.5 and E18.5). ${ }^{2}$ Three knockout mouse models were created for Recql4 to gain new information about the phenotype and the function of the gene and protein. The first mouse model lacking exons 5-8 was embryologically lethal and thus it could not be used as a model for the RECQL4 syndromes. ${ }^{36}$ Hoki et al ${ }^{37}$ created the second mouse model by deleting exon 13 of the Recql4 gene and thus disrupting the helicase domain. Only 5\% of Recql4-deficient mice survived more than 2 weeks. The mice represented several symptoms similar to human RECQL4 diseases, such as growth retardation, developmental defects and skin abnormalities, but they did not develop any malignancies. In the third mouse model, most of the helicase domain was deleted (exons 9-13); however, 84\% of the knockout mice survived until adulthood. ${ }^{38}$ These mice displayed skin and skeleton defects as well as palatal defects all of which have been seen in the RECQL4 patients.

The exact role of RECQL4 is unclear, but recent studies have provided some insights into its function. It has been shown that RECQL4 has a DNA strand-annealing activity and ssDNA can activate an ATPase function of RECQL4, ${ }^{39}$ but in contrast to other RecQ helicases RECQL4 does not possess a DNA helicase activity. ${ }^{39,40}$ A study using Xenopus oocyte extracts has shown that RECQL4 is crucial for the initiation of DNA replication. ${ }^{41}$ A search for the interacting partners of RECQL4 has led to the identification of ubiquitin ligases UBR1 and UBR2 of the N-end rule pathway, but the implication of this interaction is not yet known. ${ }^{40}$ Burks et $a l^{42}$ have shown that RECQL4 has a nuclear targeting signal in the $N$-terminus (amino acids 363-492), but localization studies of RECQL4 have shown both nuclear and cytoplasmic localization. 1,40,42,43 Additional localization studies in various human cells have shown that RECQL4 forms discrete nuclear foci and it 
Table 1 Reported patients with RECQL4 mutations

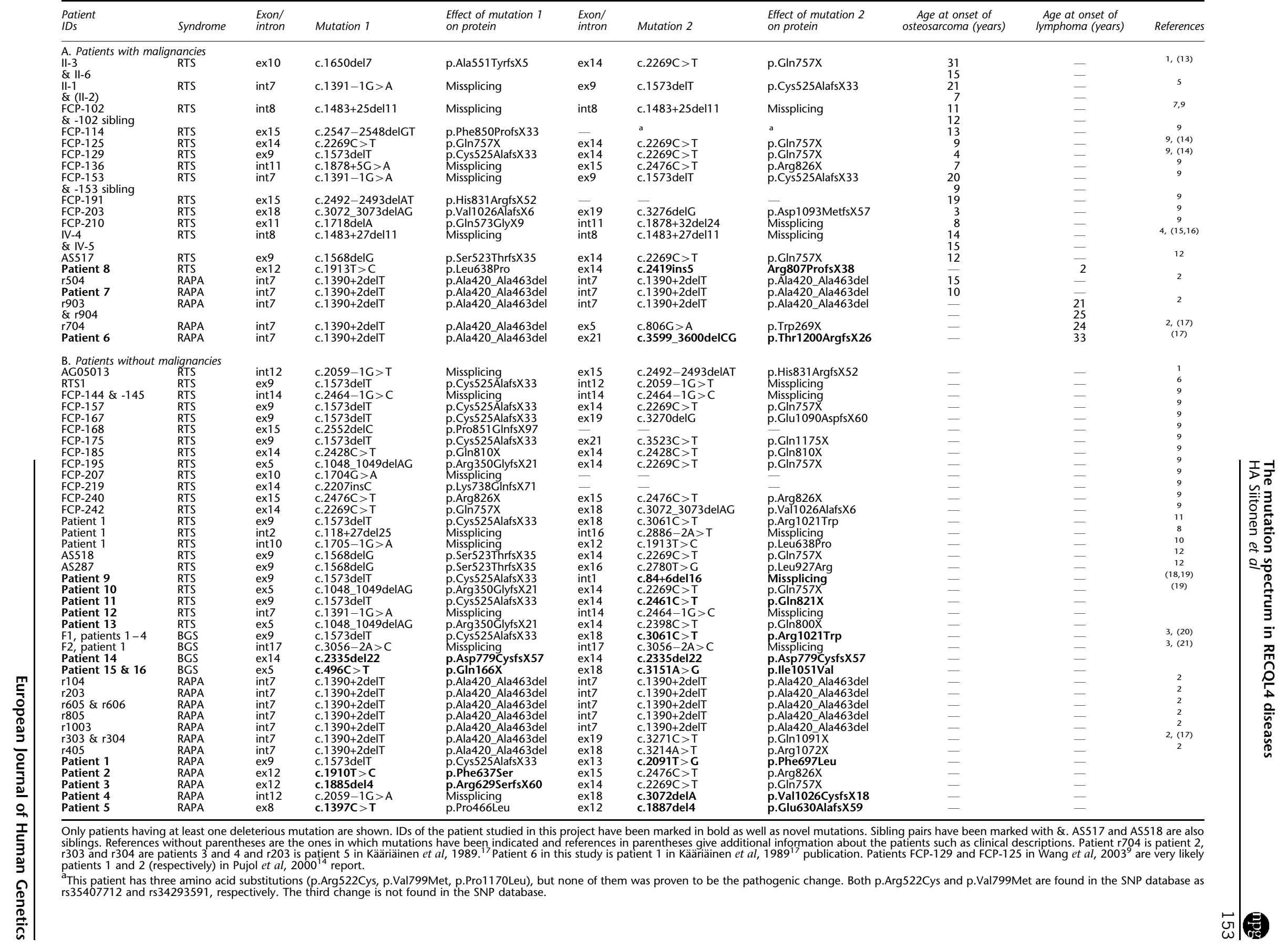


Table 2 Clinical data from 16 patients with the RECQL4 mutations

\begin{tabular}{|c|c|c|c|c|c|c|c|c|c|c|c|c|c|c|c|c|}
\hline Clinical features & $\begin{array}{c}1 \\
R A P A\end{array}$ & $\begin{array}{c}2 \\
R A P A\end{array}$ & $\begin{array}{c}3 \\
R A P A\end{array}$ & $\begin{array}{c}4 \\
R A P A\end{array}$ & $\begin{array}{c}5 \\
R A P A\end{array}$ & $\begin{array}{c}6 \\
R A P A\end{array}$ & $\begin{array}{c}7 \\
R A P A\end{array}$ & $\begin{array}{c}8 \\
R T S\end{array}$ & $\begin{array}{c}9 \\
\text { RTS }\end{array}$ & $\begin{array}{c}10 \\
R T S\end{array}$ & $\begin{array}{c}11 \\
\text { RTS }\end{array}$ & $\begin{array}{c}12 \\
\text { RTS }\end{array}$ & $\begin{array}{c}13 \\
\text { RTS }\end{array}$ & $\begin{array}{c}14 \\
B G S\end{array}$ & $\begin{array}{c}15 \\
B G S\end{array}$ & $\begin{array}{c}16 \\
B G S\end{array}$ \\
\hline Short stature $>-2 S D$ & + & + & + & + & + & + & + & + & + & + & + & - & + & + & NA & NA \\
\hline \multicolumn{17}{|l|}{ Dermatological changes } \\
\hline Poikiloderma & - & - & - & - & - & - & - & $++^{a}$ & + & + & + & + & + & + & NA & NA \\
\hline Brownish spots & - & - & + & + & - & + & + & - & - & - & - & + & - & - & NA & NA \\
\hline $\begin{array}{l}\text { Alopecia, loss of } \\
\text { eyebrows or eyelashes }\end{array}$ & - & - & - & - & - & - & - & + & - & - & - & + & + & + & NA & NA \\
\hline \multicolumn{17}{|l|}{ Skeletal abnormalities } \\
\hline Thumb a-/hypoplasia & + & + & + & + & + & + & - & + & + & + & + & - & + & + & + & + \\
\hline Radial a-/hypoplasia & + & + & + & + & + & + & - & + & + & + & + & - & + & + & + & + \\
\hline Patellar a-/hypoplasia & + & + & + & + & - & + & - & + & + & + & + & - & + & - & NA & NA \\
\hline High arched/cleft palate & + & - & + & + & - & + & - & + & + & + & + & - & + & - & NA & - \\
\hline Joint dislocations & + & - & + & - & - & - & - & + & - & - & - & + & + & + & NA & - \\
\hline Osteopenia/osteoporosis & - & - & - & - & - & - & - & - & + & + & - & + & - & + & NA & NA \\
\hline Craniosynostosis & - & - & - & - & - & - & - & - & - & - & - & - & + & + & NA & + \\
\hline \multicolumn{17}{|l|}{ Malignancies } \\
\hline Osteosarcoma & - & - & - & - & - & - & + & - & - & - & - & - & - & - & NA & NA \\
\hline Lymphoma & - & - & - & - & - & + & - & + & - & - & - & - & - & - & NA & NA \\
\hline Diarrhea & + & + & + & + & + & + & + & + & + & + & + & + & - & - & NA & NA \\
\hline Feeding problems & - & + & + & - & + & - & - & + & + & + & + & + & + & + & NA & NA \\
\hline Hearing problems, hearing loss & + & - & - & - & - & - & - & - & + & + & - & - & - & + & NA & NA \\
\hline Gender & $\mathrm{M}$ & $\mathrm{M}$ & $\mathrm{F}$ & $\mathrm{M}$ & $\mathrm{F}$ & $\mathrm{F}$ & $\mathrm{M}$ & $\mathrm{M}$ & $\mathrm{M}$ & $\mathrm{F}$ & $\mathrm{F}$ & $\mathrm{M}$ & $\mathrm{F}$ & $\mathrm{F}$ & $\mathrm{F}$ & $\mathrm{F}$ \\
\hline Age in January 2008 (years) & 23 & 5 & $5^{b}$ & 28 & 1 & 35 & 10 & $c$ & 12 & 13 & 11 & 14 & 4 & 3 & $\mathrm{~d}$ & e \\
\hline
\end{tabular}

${ }^{a}$ Atypical poikiloderma, the age of onset 2.5 years.

${ }^{\mathrm{b}}$ Patient lost for follow-up at the age of 5 years.

'Patient died at the age of 3.5 years.

dPregnancy terminated at 11.5 weeks of gestation.

epregnancy terminated at 23 weeks of gestation.

Brownish spots refer to the brown pigmentation that resembles irregularly shaped café-au-lait spots. This does not include hyperpigmentation seen in poikiloderma.

colocalizes with promyelotic leukemia protein (PML) nuclear bodies as well as with regions of ssDNA. RECQL4 also forms a complex with Rad51 and colocalizes with it in human cells after the induction of DNA double-strand breaks. $^{43}$

The aim of this study was to identify novel RECQL4 mutations in patients with clinically suspected RTS, RAPADILINO or BGS and to collect and analyze the precise clinical data from the patients with the identified mutations. We also updated the current cancer status of RAPADILINO patients and observed that both lymphoma and osteosarcoma had been diagnosed among RAPADILINO patients again confirming the association between RECQL4 mutations and cancer risk.

\section{Materials and methods}

\section{Subjects, samples and clinical data}

This study (collection of samples, the RECQL4 gene analysis and evaluation of medical records) was approved by the Ethical Committee of the Joint Authority for the Hospital District of Helsinki and Uusimaa, Finland. As the phenotype of patients carrying RECQL4 mutations can be quite variable, we accepted for the study DNA samples from all patients who were suspected to have a clinical diagnosis of RTS, RAPADILINO or BGS. After obtaining the patients' consent, the referring clinicians sent us DNA samples from a total of 35 patients from several different populations. More thorough clinical data were requested only from patients who were found to have RECQL4 mutations. Clinical data were collected from the medical records of the patients by clinicians who filled out a uniform medical questionnaire documenting the presence of features commonly associated with RTS, RAPADILINO and BGS. These features are listed in Table 2 . In addition, we reviewed medical records from 15 Finnish RAPADILINO patients to update their cancer status.

\section{Samples and mutation analysis}

The whole RECQL4 gene was sequenced including all exons and exon-intron boundaries as well as all introns except intron 12 (primer sequences are available upon request). ${ }^{2}$ Samples from patients 9 and 10 were sequenced as described by Wang et al. ${ }^{9}$ The allelic segregation of the mutations was confirmed from both parental samples in all cases except in two families. In the case of patient 
8 samples were available only from the patient and his mother. In the case of patient 12, parental samples were not available.

\section{Mutation nomenclature}

Mutation positions are given according to the Reference sequence for RECQL4 (NM_004260). Numbering starts from nucleotide 33, which is the A of the ATG-translation initiation codon.

\section{In silico analyses}

Sequences for protein sequence comparisons were retrieved from NCBI (www.ncbi.nlm.nih.gov/) and aligned using the ClustalW program (align.genome.jp/). Protein sequence entities for different species were NP_004251.2 Homo sapiens, XP_520023.2 Pan troglodytes, NP_478121.2 Mus musculus, XP_216973.4 Rattus norvegicus, XP_539222.2 Canis familiaris, NP_001091506.1 Bos taurus, XP_427538.2 Gallus gallus, NP_001089101.1 Xenopus laevis, NP_652607.1 Drosophila melanogaster, XP_315948.4 Anopheles gambiae, NP_001053140.1 Oryza sativa and NP_174109.2 Arabidopsis thaliana.

The effects of the amino acid substitutions were predicted using the PolyPhen (coot.embl.de/PolyPhen/) and SIFT (blocks.fhcrc.org/sift/SIFT.html) programs. Reference sequence NP_004251.2 (gi116812616) was used for human RECQL4.

\section{Results \\ RECQL4 mutations}

On the basis of earlier publications, 35 different mutations in the RECQL4 gene have been identified. In this study, a molecular change in both alleles of the RECQL4 gene was found in 16 out of 35 patients analyzed (46\%), and 14 of these mutations were novel. All reported patients with deleterious RECQL4 mutations are presented in Table 1. The Supplementary Table 1 shows all the identified mutations in a structural order from the $5^{\prime}$ end to the $3^{\prime}$ end of RECQL4 as well as the incidence of each mutation.

Nine of the novel mutations caused an early stop codon or a frameshift, both leading to truncated polypeptides: c.496C > T (p.Gln166X), c.1885del4 (p.Arg629SerfsX60), c.1887del4 (p.Glu630AlafsX59), c.2335del22 (p.Asp779 CysfsX57), c.2398C > T (p.Gln800X), c.2419ins5 (Arg807ProfsX38), c.2461C > T (p.Gln821X), c.3072delA (p.Val626CysfsX18) and c.3599_3600delCG (p.Thr1200ArgfsX26). Four mutations caused novel amino acid changes: c.1397C > T (p.Pro466Leu), c.1910T >C (p.Phe637Ser), c.2091T > G (p.Phe697Leu) and c.3151A > G (p.Ile1051Val). One of the identified mutations was a 16-base pair deletion in intron 1 (c.84+6del16). As previously described, RECQL4 has an unusual genomic structure with 13 out of 20 introns being less than $100 \mathrm{bp}$ in length. This intronic deletion results in an intron of $49 \mathrm{bp}$ in length that is probably too small for correct splicing. ${ }^{4,7-9}$

From the sequenced samples, we found a total of 10 amino acid substitutions. Two of these, p.Glu267Asp and p.Arg $1005 \mathrm{Gln}$, were frequently detected and they are also reported as common variants in NCBI's SNP database, with their accession numbers and allele frequencies in parentheses: rs4244612 (0.461 \pm 0.134$)$ and rs4251691 $(0.423 \pm 0.181)$, respectively. Patient 1 had two amino acid substitutions, p.Glu71Gly and p.Phe697Leu, on the same allele. As p.Glu71Gly has also been found in an earlier RTS study from patients with no other mutations in RECQL4 ${ }^{9}$ and from NCBI's SNP database (rs34642881) with allele frequency $0.067 \pm 0.170$, it could also represent a polymorphism. However, this does not rule out the possibility that the combined effect of these two mutations could be pathogenic. A similar situation was observed in patient 5 who had the p.Arg522His and p.Pro466Leu substitutions in the same allele. p.Arg522His has been reported in NCBI's SNP database (rs35842750) and both heterozygotes and homozygotes were identified in the population studies, thus suggesting this change to be a common variant. Interestingly, patients 1,9 and 11 with the p.Cys525AlafsX33 mutation had the amino acid substitution p.Ser523Thr in the same allele. This amino acid substitution is not found in the SNP database and may be specifically linked to the p.Cys525AlafsX33 mutation or represent a rare haplotype..$^{3,5,6}$

It is difficult to interpret the effects of the amino acid substitutions because there is no crystallographic model for RECQL4 and the exact physiological role of this protein is unknown. However, bioinformatics tool can be used to predict the significance of the amino acid substitution on the protein. We performed PolyPhen and SIFT analyses for the four novel amino acid substitutions, for the p.Glu71Gly and p.Arg522His changes and for the six previously published amino acid substitutions. In addition, we aligned 12 RECQL4 orthologs from different species to determine conserved amino acids. The results from these analyses are presented in Supplementary Table 2. PolyPhen and SIFT results were indicative, but sometimes conflicting as in the case of p.Pro466Leu and p.Phe697Leu. PolyPhen predicts these changes to be probably damaging in contrast to SIFT's prediction that the changes will be tolerated. These amino acids were well conserved among 12 species. The p.Phe638Pro change found in our and in a previous study ${ }^{10}$ is predicted to be damaging by both PolyPhen and SIFT and it is also evolutionarily conserved in all studied species except Anopheles. Interestingly, the novel p.Phe637Ser change in an adjacent amino acid found from a RAPADILINO patient is less conserved among species, but yet it is predicted to affect protein function. The p.Ile1051Val substitution is not predicted to affect protein function, even though it is conserved in all six mammals.

It is very likely that at least p.Pro466Leu, p.Phe637Ser and p.Phe697Leu found in this study are the second 
pathogenic mutations in these patients even though the possibility of a promoter region mutation that could lead to loss of translation of a specific allele can not be excluded. The effect of p.Ile1051Val remains unsure; however, it was the only change found from siblings 15 and 16 in addition to the p.Gln166X mutation that is clearly pathogenic. This change was not found in SNP database, but further studies will be needed to conclude whether it is pathogenic or a rare benign variant.

\section{Analysis of phenotypes associated with RECQL4 mutations}

Detailed clinical data were collected from 16 patients having RECQL4 mutations (Table 2). We were interested in clinical features that are frequently described in the literature regarding RTS, RAPADILINO and BGS patients. Short stature was a typical feature for 13 of 14 patients. When evaluating dermatological features 6 out of 14 patients had typical poikiloderma and one patient had atypical poikiloderma. One of these RTS patients also had brownish spots, which were also described in four RAPADILINO patients. Alopecia and loss of eyebrows or eyelashes was found in only four RTS patients. Thumb and radial a-/hypoplasias were diagnosed in 14 out of 16 patients, thus making it the most common feature in this cohort in addition to short stature. Diarrhea was reported in 12 out of 14 patients, but other features were found irregularly. In conclusion, the patients had a minimum of four findings, but none of them actually had all of the features listed in Table 2.

\section{Cancer status}

As RTSII patients with RECQL4 mutations are known to be particularly susceptible to osteosarcoma, we wanted to determine the cancer status among the RAPADILINO patients. On the basis of the previous study of RAPADILINO patients, we knew that patient r504 had osteosarcoma in her teens and that patient r903 had lymphoma in her early twenties. ${ }^{2}$ For this study, we collected medical records from all 15 Finnish RAPADILINO patients. Strikingly, we identified one additional osteosarcoma and three new lymphoma cases among these patients. Patient r704 and patient r903's sibling r904 developed the lymphoma in their twenties, and patient 6 in her thirties. Patient 7 developed osteosarcoma at the age of 10 years (Table $1 \mathrm{~A})$. Thus, out of 15 Finnish RAPADILINO patients there have been two diagnoses of osteosarcoma and four of lymphoma making the cancer incidence very high among Finnish RAPADILINO patients (40\%).

Cancer status was also obtained for the other patients with RECQL4 mutations in this study. One RTS patient developed lymphoma at the age of 2 years and died of it at the age of 3.5 years (patient 8 ). This patient had been diagnosed with RTS as he had poikiloderma even though it was atypical. Interestingly, none of the RTS patients in this study had developed osteosarcoma in contrast to the osteosarcoma incidence that was as high as $48 \%$ in the most extensive study evaluating cancer status among RTS patients with the RECQL4 mutations. ${ }^{9}$

\section{Discussion}

Mutation screening is a powerful diagnostic tool in syndromes where phenotypic variation is wide, such as the RECQL4 associated syndromes. At the moment, 64 patients with two RECQL4 mutations have been identified and in addition, in four patients only one deleterious mutation is known (Table 1). When reviewing all the single mutations identified in RECQL4 syndromes it can be concluded that the majority of mutations has been found in only one patient, but there are three mutations that are more prevalent (Supplementary Table 1). The most common RECQL4 mutation is c. $1390+2$ delT (p.Ala420_ Ala463del) which is enriched in the isolated Finnish population. All the Finnish RAPADILINO patients are at least compound heterozygotes for this mutation and therefore have at least one gene copy that encodes a RECQL4 protein from which 44 amino acids are missing. In addition, the c.1573delT mutation (p.Cys525AlafsX33) has been found in a total of 12 alleles and interestingly from patients with all three syndromes. The c.2269C $>$ T (p.Gln757X) mutation has been found in 10 alleles and from RTS and RAPADILINO patients. RECQL4 mutations are typically predicted to be truncating caused by either an early stop codon, missplicing or a frameshift. Over half of these mutations (Supplementary Table 1) are predicted to destroy the reading frame before or in the helicase domain (encoded by exons 8-14) that is thought to be critical for the function of RECQL4 even if the DNA helicase activity of RECQL4 has not been shown. ${ }^{39,40}$ The four amino acid substitutions located in the helicase domain may also disturb the functioning of the protein.

There is no clear genotype-phenotype correlation when comparing the phenotype conveyed by specific mutations. Truncating mutations in both alleles usually strongly suggest RTSII or BGS; however, a few RAPADILINO patients have two truncating mutations as well. In addition, amino acid substitutions have been found in patients with all three syndromes (Supplementary Table 1).

On the basis of this and previous studies, we conclude that clinical features of patients with RECQL4 mutations can be quite variable. However, it seems that approximately $85 \%$ of the patients have short stature and skeletal abnormalities, such as thumb, radial and/or patellar a-/hypoplasias. This is complicated by the fact that there is clinical variability even between siblings who carry the same mutations. When evaluating the symptoms of three brother-sister sibling pairs with RAPADILINO it was noted that the clinical picture of the brothers was 
significantly milder than their sisters' and it would have been difficult to suspect the RAPADILINO diagnosis without the sister with typical features. ${ }^{2}$

When evaluating differences among RECQL4 syndromes it seems that a poikilodermatous rash is a distinguishing feature between RTS and RAPADILINO. Thorough examination of the skin is important as the onset and distribution of poikiloderma can be atypical. Usually, poikiloderma in RTS appears at the age of 3-6 months and starts spreading from the cheeks to extremities usually sparing the trunk and abdomen. If the patient develops poikiloderma at an early age and with typical pattern of spread, this fulfills the criteria for a diagnosis of RTS. ${ }^{25}$ If the patient has RECQL4 mutations, but no evidence of poikiloderma, the diagnosis is more likely RAPADILINO syndrome. As seen in Table 1 most patients (63\%) have the RTS diagnosis whereas approximately $30 \%$ of cases are RAPADILINO patients and fewer than $10 \%$ of cases have BGS.

From the reported patients with the RECQL4 mutations $37 \%$ have developed malignancies (Table 1). Interestingly, in six out of seven sibling pairs both siblings have developed malignancies thus suggesting that genetic background has a high impact on cancer risk. Osteosarcomas are typical for RTS patients with RECQL4 mutations, whereas emphasized here RAPADILINO patients are at risk for both lymphomas and osteosarcomas. Although the number of patients is small, given the low incidence of osteosarcoma and lymphoma in the general population the finding of two cases of osteosarcoma and four cases of lymphoma in 15 patients demonstrate a clear susceptibility to these malignancies. On the basis of the existing data from the function of RECQL4 it is not possible to explain why the Finnish RAPADILINO patients are susceptible to developing both lymphoma and osteosarcoma. However, there may be a connection between the cancer and an abnormal localization of the RECQL4 protein encoded by the most common RECQL4 mutation (c.1390+2delT/ p.Ala420_Ala463del). On account of the mutation, the domain that is needed for a nuclear retention of RECQL4 is missing and probably because of this the localization of defective RECQL4 is cytoplasmic. ${ }^{42}$ It is also possible that other genetic loci may modify cancer risk, but these questions remain open.

Interestingly, among the 100 knockout mice (lacking exons 9-13) five developed cancers of which three were lymphomas and two were osteosarcomas. In addition, the Recql4 ${ }^{-/-}, \mathrm{ApC}^{\mathrm{Min} /+}$ mice had a two-fold increase in the multiplicity of macroadenomas locating in the GI tract and large intestine and macroadenomas were also larger in size. ${ }^{38}$ Additional analyses of these mice might shed light on cancers developed in human RECQL4 defective patients.

In conclusion, the identification of RECQL4 mutations is significant as it clarifies the risk of the recurrence in the family and reveals the increased cancer risk. The parents of patients with RECQL4 mutations need to be advised to pursue counseling and regular follow-up sessions for their children. It is very important to note that the follow-up needs to be long-term as the age at onset of cancer can be very variable being from 2 to 33 years among the reported patients with RECQL4 mutations (Table 1). Clinicians should be aware of both osteosarcoma and lymphoma risk when following patients with the RECQL4 mutations and counsel their patients accordingly until more experience accumulates.

\section{Acknowledgements}

Ritva Timonen and Katriina Hautaviita are acknowledged for excellent technical help. Jonna Tallila, Heli Honkala, Heidi Nousiainen and Juha Kere are warmly thanked for the critical reading of the manuscript. This study has been funded by the Helsinki Graduate School in Biotechnology and Molecular Biology (HAS) and by the Finnish Cancer Organizations (MK, HK, HAS). Additional funding included the National Institutes of Health (HD024064, BCMMRDDRC (LLW, SEP) and NICHD KO8HD42136 (LLW), and the Doris Duke Charitable Foundation (LLW).

\section{References}

1 Kitao S, Shimamoto A, Goto M et al: Mutations in RECQL4 cause a subset of cases of Rothmund-Thomson syndrome. Nat Genet 1999; 22: 82-84.

2 Siitonen $\mathrm{HA}$, Kopra $\mathrm{O}$, Kääriäinen $\mathrm{H}$ et al: Molecular defect of RAPADILINO syndrome expands the phenotype spectrum of RECQL diseases. Hum Mol Genet 2003; 12: 2837-2844.

3 Van Maldergem L, Siitonen HA, Jalkh N et al: Revisiting the craniosynostosis-radial ray hypoplasia association: Baller-Gerold syndrome caused by mutations in the RECQL4 gene. J Med Genet 2006; 43: 148-152.

4 Balraj P, Concannon P, Jamal R et al: An unusual mutation in RECQ4 gene leading to Rothmund-Thomson syndrome. Mutat Res 2002; 508: 99-105.

5 Lindor NM, Furuichi Y, Kitao S, Shimamoto A, Arndt C, Jalal S: Rothmund-Thomson syndrome due to RECQ4 helicase mutations: report and clinical and molecular comparisons with Bloom syndrome and Werner syndrome. Am J Med Genet 2000; 90: $223-228$.

6 Beghini A, Castorina P, Roversi G, Modiano P, Larizza L: RNA processing defects of the helicase gene RECQL4 in a compound heterozygous Rothmund-Thomson patient. Am J Med Genet A 2003; 120: 395-399.

7 Wang LL, Worley K, Gannavarapu A, Chintagumpala MM, Levy ML, Plon SE: Intron-size constraint as a mutational mechanism in Rothmund-Thomson syndrome. Am J Hum Genet 2002; 71: $165-167$.

8 Broom MA, Wang LL, Otta SK et al: Successful umbilical cord blood stem cell transplantation in a patient with RothmundThomson syndrome and combined immunodeficiency. Clin Genet 2006; 69: 337-343.

9 Wang LL, Gannavarapu A, Kozinetz CA et al: Association between osteosarcoma and deleterious mutations in the RECQL4 gene in Rothmund-Thomson syndrome. J Natl Cancer Inst 2003; 95: 669-674.

10 Sznajer Y, Siitonen HA, Roversi G et al: Atypical RothmundThomson syndrome in a patient with compound heterozygous mutations in RECQL4 gene and phenotypic features in RECQL4 syndromes. Eur J Pediatr 2008; 167: 175-181. 
11 Kellermayer R, Siitonen HA, Hadzsiev K, Kestilä M, Kosztolanyi G: A patient with Rothmund-Thomson syndrome and all features of RAPADILINO. Arch Dermatol 2005; 141: 617-620.

12 Cabral RE, Queille S, Bodemer C et al: Identification of new RECQL4 mutations in Caucasian Rothmund-Thomson patients and analysis of sensitivity to a wide range of genotoxic agents. Mutat Res 2008 [e-pub ahead of print].

13 Lindor NM, Devries EM, Michels VV et al: Rothmund-Thomson syndrome in siblings: evidence for acquired in vivo mosaicism. Clin Genet 1996; 49: 124-129.

14 Pujol LA, Erickson RP, Heidenreich RA, Cunniff C: Variable presentation of Rothmund-Thomson syndrome. Am J Med Genet 2000; 95: 204-207.

15 Tong M: Rothmund-Thomson syndrome in fraternal twins. Pediatr Dermatol 1995; 12: 134-137.

16 Miozzo M, Castorina P, Riva P et al: Chromosomal instability in fibroblasts and mesenchymal tumors from 2 sibs with Rothmund-Thomson syndrome. Int J Cancer 1998; 77: 504-510.

17 Kääriäinen H, Ryöppy S, Norio R: RAPADILINO syndrome with radial and patellar aplasia/hypoplasia as main manifestations. Am J Med Genet 1989; 33: 346-351.

18 Kant SG, Baraitser M, Milla PJ, Winter RM: Rapadilino syndrome - a non-Finnish case. Clin Dysmorphol 1998; 7: 135-138.

19 Hilhorst-Hofstee Y, Shah N, Atherton D, Harper JI, Milla P, Winter RM: Radial aplasia, poikiloderma and auto-immune enterocolitis - new syndrome or severe form of Rothmund-Thomson syndrome? Clin Dysmorphol 2000; 9: 79-85.

20 Van Maldergem L, Verloes A, Lejeune L, Gillerot Y: The Baller-Gerold syndrome. J Med Genet 1992; 29: 266-268.

21 Megarbane A, Melki I, Souraty N et al: Overlap between Baller-Gerold and Rothmund-Thomson syndrome. Clin Dysmorphol 2000; 9: 303-305.

22 Rothmund A: Über Cataracten in Verbindung mit einer eigentumlichen Hautdegeneration. Arch Klin Exp Ophtal 1898; 4: $159-182$

23 Thomson MS: Poikiloderma congenitale. Br J Dermatol 1936; 4: $221-234$.

24 Taylor WB: Rothmund's syndrome; Thomson's syndrome; congenital poikiloderma with or without juvenile cataracts. AMA Arch Derm 1957; 75: 236-244.

25 Wang LL, Levy ML, Lewis RA et al: Clinical manifestations in a cohort of 41 Rothmund-Thomson syndrome patients. Am J Med Genet 2001; 102: 11-17.

26 Jam K, Fox M, Crandall BF: RAPADILINO syndrome: a multiple malformation syndrome with radial and patellar aplasia. Teratology 1999; 60: 37-38.

27 Vargas FR, de Almeida JC, Llerena Junior JC, Reis DF: RAPADILINO syndrome. Am J Med Genet 1992; 44: 716-719.

28 Gripp KW, Stolle CA, McDonald-McGinn DM et al: Phenotype of the fibroblast growth factor receptor 2 Ser351Cys mutation: Pfeiffer syndrome type III. Am J Med Genet 1998; 78: 356-360.
29 Gripp KW, Stolle CA Celle L, McDonald-McGinn DM, Whitaker LA, Zackai EH: TWIST gene mutation in a patient with radial aplasia and craniosynostosis: further evidence for heterogeneity of Baller-Gerold syndrome. Am J Med Genet 1999; 82: $170-176$.

30 Galea P, Tolmie JL: Normal growth and development in a child with Baller-Gerold syndrome (craniosynostosis and radial aplasia). J Med Genet 1990; 27: 784-787.

31 Cohen Jr MM, Toriello HV: Is there a Baller-Gerold syndrome? Am J Med Genet 1996; 61: 63-64.

32 Kitao S, Ohsugi I, Ichikawa K, Goto M, Furuichi Y, Shimamoto A: Cloning of two new human helicase genes of the RecQ family: biological significance of multiple species in higher eukaryotes. Genomics 1998; 54: 443-452.

33 Sharma S, Doherty KM, Brosh Jr RM: Mechanisms of RecQ helicases in pathways of DNA metabolism and maintenance of genomic stability. Biochem J 2006; 398: 319-337.

34 Bachrati CZ, Hickson ID: RecQ helicases: guardian angels of the DNA replication fork. Chromosoma 2008; 117: 219-233.

35 Hanada K, Hickson ID: Molecular genetics of RecQ helicase disorders. Cell Mol Life Sci 2007; 64: 2306-2322.

36 Ichikawa K, Noda T, Furuichi Y: [Preparation of the gene targeted knockout mice for human premature aging diseases, Werner syndrome, and Rothmund-Thomson syndrome caused by the mutation of DNA helicases]. Nippon Yakurigaku Zasshi 2002; 119: 219-226.

37 Hoki Y, Araki R, Fujimori A et al: Growth retardation and skin abnormalities of the Recql4-deficient mouse. Hum Mol Genet 2003; 12: 2293-2299.

38 Mann MB, Hodges CA, Barnes E, Vogel H, Hassold TJ, Luo G Defective sister-chromatid cohesion, aneuploidy and cancer predisposition in a mouse model of type II Rothmund-Thomson syndrome. Hum Mol Genet 2005; 14: 813-825.

39 Macris MA, Krejci L, Bussen W, Shimamoto A, Sung P: Biochemical characterization of the RECQ4 protein, mutated in Rothmund-Thomson syndrome. DNA Repair (Amst) 2006; 5: $172-180$.

40 Yin J, Kwon YT, Varshavsky A, Wang W: RECQL4, mutated in the Rothmund-Thomson and RAPADILINO syndromes, interacts with ubiquitin ligases UBR1 and UBR2 of the N-end rule pathway. Hum Mol Genet 2004; 13: 2421-2430.

41 Sangrithi MN, Bernal JA, Madine $\mathrm{M}$ et al: Initiation of DNA replication requires the RECQL4 protein mutated in RothmundThomson syndrome. Cell 2005; 121: 887-898.

42 Burks LM, Yin J, Plon SE: Nuclear import and retention domains in the amino terminus of RECQL4. Gene 2007; 391: 26-38.

43 Petkovic M, Dietschy T, Freire R, Jiao R, Stagljar I: The human Rothmund-Thomson syndrome gene product, RECQL4, localizes to distinct nuclear foci that coincide with proteins involved in the maintenance of genome stability. J Cell Sci 2005; 118: $4261-4269$.

Supplementary Information accompanies the paper on European Journal of Human Genetics website (http://www.nature.com/ejhg) 